\title{
The Accessible Interactive Mode of Traditional Printing Based on Two-Dimensional Code
}

\author{
Lu-Na Mao, Xiao-Jie Huang, Zhi Yu, Wei Wang, Sheng Zhou \\ Zhejiang Provincial Key Laboratory of Service Robot, College of Computer Science, Zhejiang University, Hangzhou \\ 310027, China \\ E-mail: lunanar@zju.edu.cn, huangxiaojie@zju.edu.cn, yuzhirenzhe@zju.edu.cn,wangwei_eagle@zju.edu.cn, \\ zhousheng_zju@zju.edu.cn
}

\begin{abstract}
There is a serious barrier to the interaction between the visually impaired and traditional printing, so this paper proposed a new accessible interaction on the basis of the existing application Voiceye. The whole process is uploading editable text information that corresponds to paper material to the cloud firstly, then scanning the two-dimensional code with the mobile terminal to help users get text online which could be broadcast with voice. Compared with Voiceye, which storing the whole text, the two-dimensional code generated in this paper only contains URL of the text instead, leading to much less information and higher fault tolerance. This interaction is of great significance to widen access to information to the visually impaired people.
\end{abstract}

Keywords- interaction; visually impaired people; twodimensional code

\section{INTRODUCTION}

The development of science and technology leads to huge changes of the way of information interaction in our daily life, more and more traditional paper prints have been replaced by virtual information media in the Internet constantly. Printed materials, however, are still an important link in information dissemination, acting as information carrier in our daily life with virtual information media. For ordinary people, the paper prints and virtual information media can provide rich information in the form they are good at respectively and be accessed successfully by people, for the special groups; however, they behave not so well, especially for visually impaired people.

Visually impaired people are those whose visual organs or visual neurons in the brain become diseased, they have different degrees of vision loss [1]. Visually impaired people is a special group in society, many problems that ordinary people cannot imagine exist in the aspects of their life, study, travel and employment, but at the same time they also need access to health, employment and social welfare and other aspects of information. Our country has more than 10 million blind people and low-vision population are about three times that of the blind, blind people who received education accounts for only about $1.5 \%-3 \%$ of the total and less than 300000 people[2], this number will become bigger and bigger with the development of the social aging phenomenon [3]

Nowadays the interactive mode between the visually impaired people and the traditional publications is mainly braille, however braille publications have the characteristics of high cost, long cycle and slow renewal iteration, and it is gradually not suitable for the information age with exponential growth of information. The current way of reading for the visually impaired people in our country mainly includes three ways: visual reading, touch reading, hearing read [4]. How to propose a new interaction pattern to broaden the access to information for visually impaired people is a problem to be solved.

In order to solve this problem, some solutions both at home and abroad have been put forward. Tsinghua University in 2001 started to develop a braille display (braille) touch screen, it makes "text" up or down to show braille by adopting a new material of piezoelectric ceramics, and the visually impaired people interact with computer by touch, and read the information on the computer by touch. Jun-ying Yang et al. proposed accessible digital library in 2011, which can provide users with all kinds of books, periodicals and audio-visual materials, people can remote login and use the library [5]. Oppenheim C and K, Selby pointed out in the paper[6] that in order to solve the website accessibility, companies such as Yahoo and AltaVista have made some methods and specifications to enable the visually impaired people to browse the website for information. Craven $\mathrm{J}$ also pointed out in his survey [7] that visually impaired people spend more time on simple web information retrieval than the general population, and it will be more convenient to search for information for visually impaired people if the site has better consistency and simpler design module. This is consistent with the study results of Oppenheim C and Selby K. To use accessible digital library [8], however, the first thing must be solved is the portal of the blind, namely, the design of a website should facilitate the operation of the blind such as page layout should consider the consistency and continuity, although accessible digital library has been developed, only a tiny fraction of visually impaired people use it in fact. And it helps little for the accessible interaction mode of traditional paper prints.

Based On the accessible interaction mode of traditional paper prints, South Korean Voiceye Company creatively develops the mobile client applications to help visually impaired people to read, using two-dimensional code scanning technology, the application saves the content of two pieces of A4 paper size in $2.5 \mathrm{~cm} * 2.5 \mathrm{~cm}$ square code which is printed along with the text body. The visually 
impaired can use the mobile client application to scan the two-dimensional code in the printing materials, and gain the text information, and then according to need display or voice broadcast the text. It is of important significance to break through the interaction mode between traditional printing materials and the visually impaired people, and win an ICT Special Educational Needs Solution prize in 2012 at annual BETT conference which known as "Oscar" education software, information files of some governments or public authorities in South Korea embedded Voiceye two-dimensional code are printed and released.

However, the mobile application still has more shortage: because all the information in the A4 page is compressed down to a $2.5 \mathrm{~cm} * 2.5 \mathrm{~cm}$ space, huge amount of information leads a dense dot matrix in two-dimensional code, and the print quality must be very high to ensure good scan results, so the cost is higher, and the size of information can be saved is still limited. At the same time, because of the dense and informative dot matrix in the twodimensional code, mobile phones should scan the code at a precise position. Thus Voiceye Company has produced brackets for mobile phones to scan two-dimensional code, and strictly regulates the location of the printed twodimensional code to ensure good scan effect. Yet this is not convenient for visually impaired people to carry and use, and for the real condition of the use of visually impaired people, the optimal scanning position cannot be perfectly aligned at any time, wear and deformation of the bracket and printing also bring challenges for practical effect for a long time.

This paper has put forward an improvement based on Voiceye, which proposes a new accessible interaction mode: first of all, considering the information capacity stored in the two-dimensional code, we store the text information on the server side, and just save the corresponding URL in two-dimensional code, so the length of the article will not be a limit; Second, due to the reduction of amount of information in the dimensional code size, scanning precision is required not so high as before, and the rate of fault tolerance of scanning code is improved, and printing quality requirements for the two-dimensional code are fewer, and it enables users to get rid of unnecessary auxiliary bracket; Finally, although reading and sharing the two-dimensional code is very convenient, people can't directly read information in it, which exits risks that it will link to illegal websites or bad contents[9] and user's private information will be filched by false twodimensional scanning, so this paper puts forward that the two-dimensional code should be encrypted, and guarantee the safety of the process that users scan the code.

\section{RESEARCH APPROACH}

The paper works to extend the interaction mode between traditional printing and the visually impaired people, and draws lessons from Voiceye work, hoping to help visually impaired people get information from printings through the two-dimensional code scanning technology, the main challenges are:

\section{A Mode of Access to Information}

The traditional way for visually impaired people to access to information is often done by touching braille to read, along with the development of the Internet, now screen readers can convert text to speech, so this system will consider what kind of information carrier we should convert text to eventually to facilitate visually impaired people accessing to information.

\section{B Capacity of Information Stored in the Two-Dimensional Code}

Because of the information capacity stored in the twodimensional code is related to the size, data coding and error correction level of the two-dimensional, so it is necessary to consider the information capacity stored in the two-dimensional code. General two-dimensional code stores text data and requires a larger code size, a big twodimensional code can affect ordinary people's reading experience, and it will lead to a capacity limit, that is to say, it can't support a long text, this is an inevitable problem that we should consider when using the two-dimensional code which stores text data.

\section{Scanning Speed and Precision}

As the users are visually impaired, in the absence of physical hardware, such as brackets for fixing mobiles, how to guarantee the angle and precision of scanning code for the visually impaired people is a key problem to be considered. Because of the user's vision problems, the first thing needs to be determined is the approximate location of two-dimensional code, secondly, it should be solved that how to locate the code and improve the scanning precision to make the scanning as convenient as possible.

\section{Information Security}

As the two-dimensional code is more and more popular, the use of two-dimensional codes to steal information is becoming more and more diverse, so to ensure the security of the information stored in the code is becoming more and more important. Information stored in two-dimensional code involves the government documents and all kinds of printed materials in which many files are confidential, other equipment except the system should not be allowed to get the information in the two-dimensional code. So it is necessary to encrypt information that is stored in the twodimensional code and guarantee the security of data.

\section{E Reading or Stop Reading Conveniently}

Reading for visually impaired people is more difficult than that for the average person, so the speed of reading are generally slower, reading and understanding a copy of the print may take a longer time, so how to allow visually impaired people to read and stop conveniently, and continue to read from last reading progress should be considered.

\section{$F \quad$ The Content of Previous or Next Page Conveniently}

Scanning code can only access to the content of current page document, how to use simple and convenient method 
to get the content of previous or next page document should be considered.

On this basis, specific solutions the paper proposes are as follows:

Visually impaired people can get text information in publications by touching and hearing, namely by the braille and voice, the voice is the most convenient and can be handled easily, so we consider converting published books into speech and expand the interaction mode between visually impaired people and publications. And voice information can be read by screen readers, and the text that need to be read can be stored in local devices or remote server, if it is stored in the local devices, general mobile storage capacity is limited which is not conducive to larger text storage and later edit after download, so this paper will store electronic text in the server.

As electronic text is stored on the server, how to obtain it by users has become a problem. Two-dimensional code technology has developed rapidly, now it is widely used in various aspects, it has advantages of low cost and mobile information. So we use two-dimensional code to store the information of text, visually impaired people get information through scanning the two-dimensional code. Considering the difficulties visually impaired people may have, improving the accuracy of code scanning is important, the accuracy relates to the speed, precision and two-dimensional code size and pixel, so the size of the twodimensional code should be modest and the content it stores cannot too much, which will greatly improve the accuracy of code scanning. At the same time, the two-dimensional code stores the URL address of text instead of text content to support long text.

And the safety of the two-dimensional code for visually impaired people is also a problem, now there exits ones who make the use of users' access to two-dimensional code which links to a malicious web site to spread the Trojan virus, or ones who steal users' private information by the two-dimensional code, and so on, so this paper considers the encryption of two-dimensional code, only specific software can decrypt information correctly by code scanning.

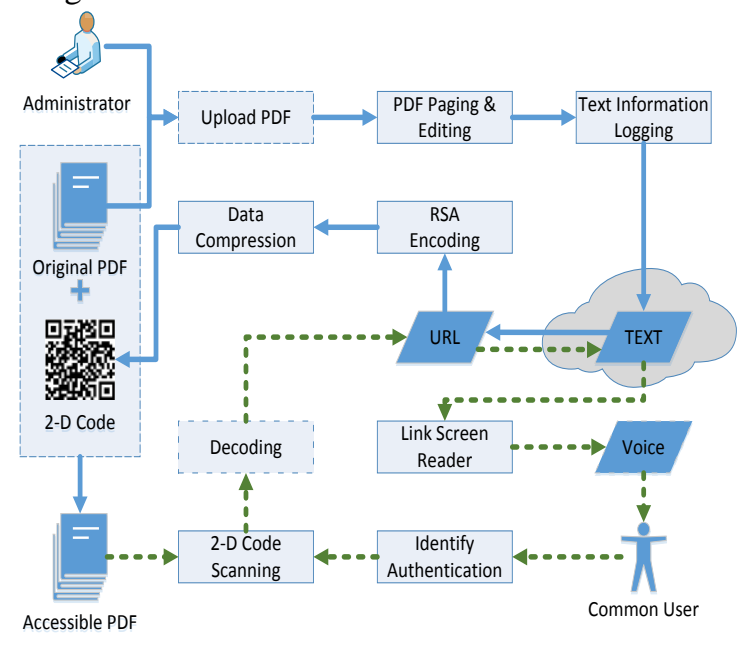

Figure 1. Overall framework of the system.
Therefore, this paper proposes a scheme that the information of each page text is stored in the cloud, and the page only saves encrypted URL, on the premise of guarantee the information security it improves the rate of fault tolerance of code scanning. The overall framework is shown in figure 1:

\section{SYSTEM ARCHITECTURE AND THE ACTUAL EFFECT}

Server-side development: we use the framework of the combination of Tomcat and MySQL to build the server side, and provide the administrator with the functions such as authentication, uploading files, editing, entering text information, generating a two-dimensional code, and so on, and the actual effect is shown in figure $2 \& 3$ :

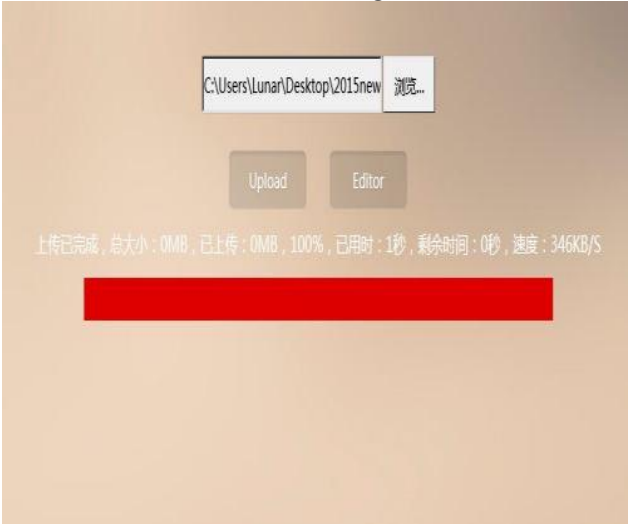

Figure 2. Example of uploading files on the server.

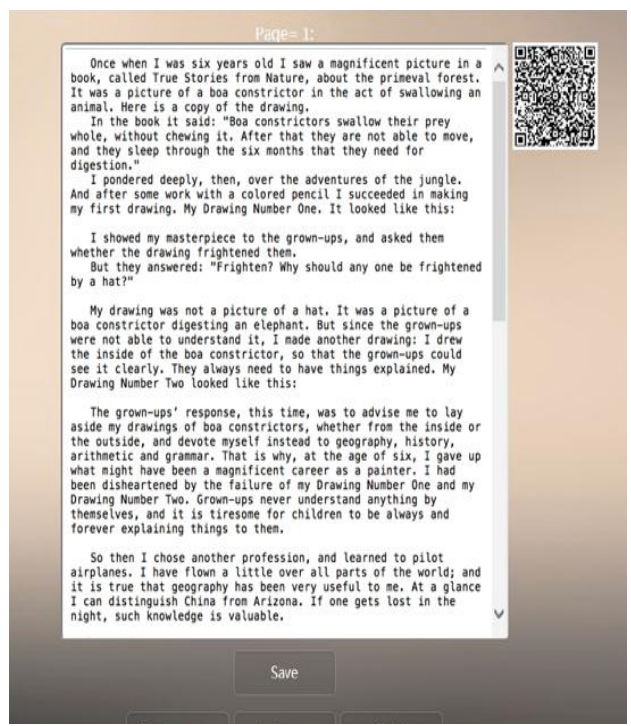

Figure 3. Example of text editing and generating a two-dimensional code.

At the same time, the server side uses the Xpdf open source projects to help quickly get the editable text in PDF, and encrypts the URL address information stored in the two-dimensional code by using the RSA [10] encryption algorithm. Due to the poor support of Java development environment for Chinese, this paper optimizes the coding 
and decoding of Chinese in the server accordingly. The details for whole processing as follow:

\section{A Rsa Encryption}

Firstly, encrypt the URL address with RSA algorithm. RSA algorithm separates keys and algorithms, making it easier to distribute keys; what's more, the keys of decryption and encryption are different, solving the problem of keys management, so confidential communications can be established without exchange of keys before. RSA are more secure than other encryption algorithms, its security depends on the decomposition of large numbers.

\section{B Data Compression}

The amount of information is increasing dramatically after a power of computing using RSA encryption, resulting in the storage in the two-dimensional code is increasing correspondingly, which reduces the resolution of the twodimensional code, so the data compression without loss of information is necessary.

In this paper, we used binary conversion. Convert the data which is decimal number after RSA encryption into 256-number-system, in order to reduce the number of bits of data, make it easier to manage and storage. Spaces will be added between remainders and "/" will be added between characters in order to decryption easier and prevent forgery and fraudulent of information. The reason for we choose 256-number-system is that the bits of remainder divided by 256 is not more than three, and the bits of the remainder is two generally, so the bits of data stored are no more than seven, which is optimum.
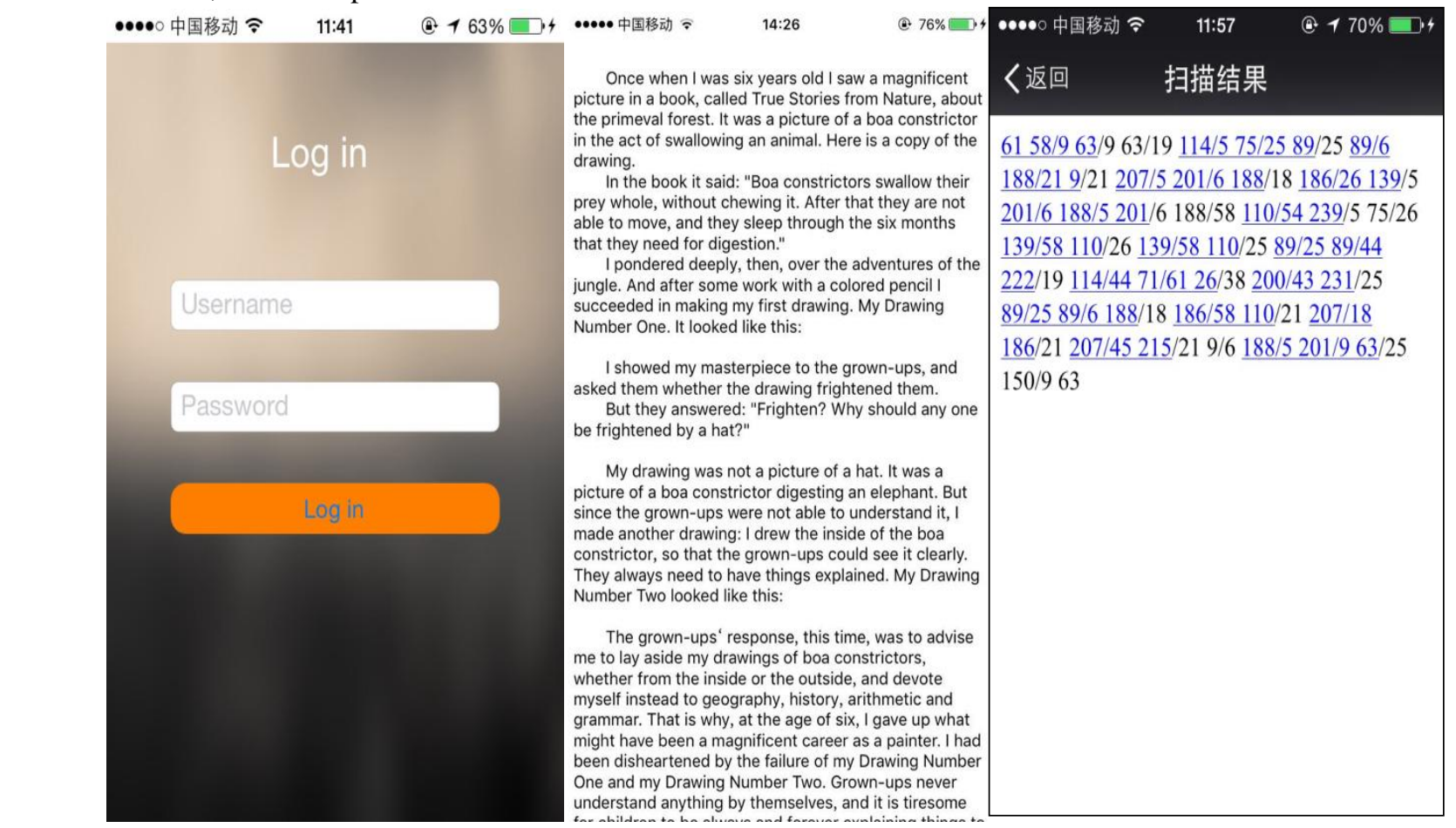

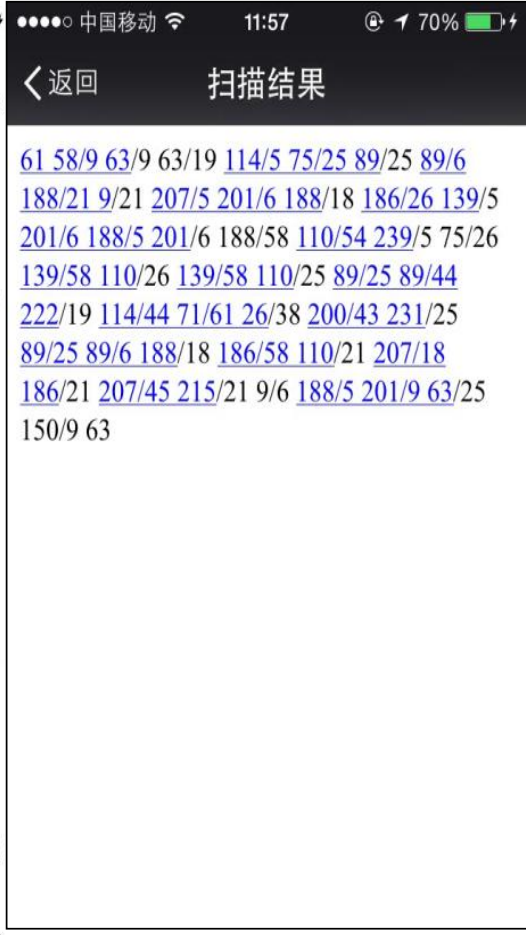

Figure 4. Example of user login interface in mobile terminal application (left), text display after scanning code correctly(mid), text display with other code-scanning tools(right).

\section{Decompression}

First convert cipher text from 256-number-system to decimal.

\section{Decryption}

Decrypt every bit of data using formula $M=c^{d} \bmod n$ Decoding principle is as follows:

$\because \quad c^{d} \equiv n^{\text {ed }}(\bmod n)$, ed $=1(\bmod p-1)$, ed $=$ $1(\bmod q-1)$

For Euler's theorem,

$\therefore \mathrm{n}^{\mathrm{ed}} \equiv \mathrm{n}(\bmod \mathrm{p}), \mathrm{n}^{\mathrm{ed}} \equiv \mathrm{n}(\bmod \mathrm{q})$

$\therefore \mathrm{n}^{\mathrm{ed}} \equiv \mathrm{n}(\bmod \mathrm{pq})$

Euler's theorem states that, if $\mathrm{n}$ a is a positive integer, and are relatively prime, then

$$
\alpha^{\Phi(\mathrm{n})} \equiv 1(\bmod \mathrm{n})
$$

Mobile terminal development: the paper uses the Objective-C language and Xcode development tools to develop the application of app based iOS operating system, the actual effect is shown in figure 4 and table 1:

Our mobile application adopts an open source code scanning tools called ZBar, and uses the Voiceover [11] in iOS system to convert text to speech, at the same time it provides the function of the paging up and down automatically or with gestures.

Due to the small capacity of information stored in the two-dimensional code, the fault tolerance rate is very high, on the premise of no vision support, even if not perfectly aligned with the precise location, it can also quickly get the correct response, and thus it has a better operation experience compared with Voiceye. 
TABLE I. THE COMPARISON BETWEEN VOICEYE AND OUR SYSTEM

\begin{tabular}{|c|c|c|c|c|}
\hline Method & $\begin{array}{c}\text { Information } \\
\text { Security }\end{array}$ & $\begin{array}{c}\text { Scanning speed } \\
\text { and precision }\end{array}$ & $\begin{array}{c}\text { Information } \\
\text { capacity }\end{array}$ & $\begin{array}{c}\text { Read } \\
\text { convenience }\end{array}$ \\
\hline Our System & $\begin{array}{c}\text { Encryption } \\
\text { Algorithm } \\
\text { precision } \\
\text { and fast } \\
\text { speed }\end{array}$ & $\begin{array}{c}\text { Cloud } \\
\text { storage, } \\
\text { support } \\
\text { long article }\end{array}$ & $\begin{array}{c}\text { Stop as you } \\
\text { like, a series } \\
\text { of gestures }\end{array}$ \\
\hline Voiceye & $\begin{array}{c}\text { No } \\
\text { encryption }\end{array}$ & $\begin{array}{c}\text { Require } \\
\text { stents }\end{array}$ & $\begin{array}{c}\text { Local } \\
\text { storage, } \\
\text { don't } \\
\text { support } \\
\text { long article }\end{array}$ & $\begin{array}{c}\text { Don't have } \\
\text { many } \\
\text { gestures }\end{array}$ \\
\hline
\end{tabular}

\section{CONCLUSION}

This paper explores a service platform for visually impaired people interact with traditional paper print which uploads editable text information in paper materials to the cloud and uses mobile terminal to scan code to get text and voice broadcast online, and thus solves the problems the visually impaired people met with when they read traditional books. Compared with the best products such as Voiceye which stores full text in the two-dimensional code in current industry, this paper generated two-dimensional codes which only contains URL, so the two-dimensional code has the advantage of less information and higher fault rate which have optimized the printing cost and user actual operation experience, the visually impaired can page up and down conveniently using the mobile application.

The actual building of the system has completed the required basic functions in this paper, but there is still some room to be improved. It can be considered to link the website and some mainstream media, directly importing documents can also be considered so that uploading documents will be not necessary, this paper uses the Voiceover as screen reader, other voice software which can interact with the user by voice can be considered and it can help visually impaired people to input voice more easily.

\section{ACKNOWLEDGEMENT}

This work is supported by National Key Technology R\&D Program (Grant No. 2014BAK15B02)

\section{REFERENCES}

[1] Xiao Meng, Xiangdong Liu. Research on one-dimensional multicolor barcode coding[J]. Journal of Zhejiang University: Engineering Science, 2004, 38(5): 559-561.

[2] Jiaqi Chen. Reflections on setting up a reading room for the blind in public libraries [J].Books and Information,2008(3).

[3] Xiaojuan Tang, Zhenxiang Sun. Research on designing strategy of blind digital library [J].Computer Knowledge and Technology: Academic Exchange, 2009, 5(11): 8689-8690.

[4] Jun Li. From paper touch reading to human-computer interactive rading development trend of reading for the blind[J].Journal of Sichuan library, 2012, (1):68-71. DOI:10.3969/j.issn.10037136.2012.01.019.

[5] Junying Yang, Linjing Zhao, Hong Zhen. Thinking on the construction of accessibility digital library from information requirement of visually impaired[J].Library Circle, 2011, (2):41-43. DOI:10.3969/j.issn. 1005-6041.2011.02.014.

[6] Oppenheim C, Selby K. Access to information on the World Wide Web for blind and visually impaired people[C]//Aslib Proceedings. MCB UP Ltd, 1999, 51(10): 335-345.

[7] Craven J. Access to electronic resources by visually impaired people[J]. Information research, 2003, 8(4): 156.

[8] Lilia Pavlova-Draganova, Desislava Paneva-Marinova, Radoslav Pavlov, et al. On the Wider Accessibility of the Valuable Phenomena of Orthodox Iconography through Digital Library[C]// International Conference Dedicated on Digital Heritage. 2010.

[9] Wei Han. Application and security analysis of mobile twodimensional code $[\mathrm{J}][\mathrm{J}]$. Information and Computer, 2012, 7: 012.

[10] Guruswami V, Sudan M. Improved decoding of Reed-Solomon and algebraic-geometric codes[C]//Foundations of Computer Science, 1998. Proceedings. 39th Annual Symposium on. IEEE, 1998: 28-37.

[11] Leporini B, Buzzi M C, Buzzi M. Interacting with mobile devices via VoiceOver: usability and accessibility issues[C]//Proceedings of the 24th Australian Computer-Human Interaction Conference. ACM, 2012: 339-348 